\title{
Investigation of the transient receptor potential (TRP) channel gene expressions in metabolic syndrome
}

\author{
Suzan Taburl, Serdar Oztuzcu², IrfanVeysel Duzen³, Ayten Eraydin', Secil Eroglu², Mesut Ozkayal, Abdullah T. Demiryürek ${ }^{4}$
}

${ }^{1}$ Division of Endocrinology, Department of Internal Medicine, Faculty of Medicine, University of Gaziantep, Gaziantep, Turkey

${ }^{2}$ Department of Medical Biology, Faculty of Medicine, University of Gaziantep, Gaziantep, Turkey

${ }^{3}$ Department of Cardiology, 25 Aralık State Hospital, Gaziantep, Turkey

${ }^{4}$ Department of Medical Pharmacology, Faculty of Medicine, University of Gaziantep, Gaziantep, Turkey

\section{Objectives:}

\section{Methods:}

Metabolic syndrome (MetS) is correlated with increased cardiovascular risk and characterized by several factors, including visceral obesity, hypertension, dyslipidemia, and insulin resistance. Several members of a large family of nonselective cation channels, e.g., transient receptor potential (TRP) channels, have been associated with the development of cardiovascular diseases. Thus, changes of TRP channel expression may account for the observed increased cardiovascular risk in MetS patients. The aim of this study was to investigate the possible contribution of TRP channel gene expressions in MetS.

A total of 54 patients with obesity-related MetS, and 41 healthy control subjects with similar age and sex were included to this study. mRNA from blood samples was extracted, and real time polymerase chain reaction on the BioMark HD dynamic array system (Fluidigm, South San Francisco, CA, USA) was performed for the TRP channel gene expressions. For calculation of the significance of differences in gene expressions, the Mann-Whitney U-test was used.
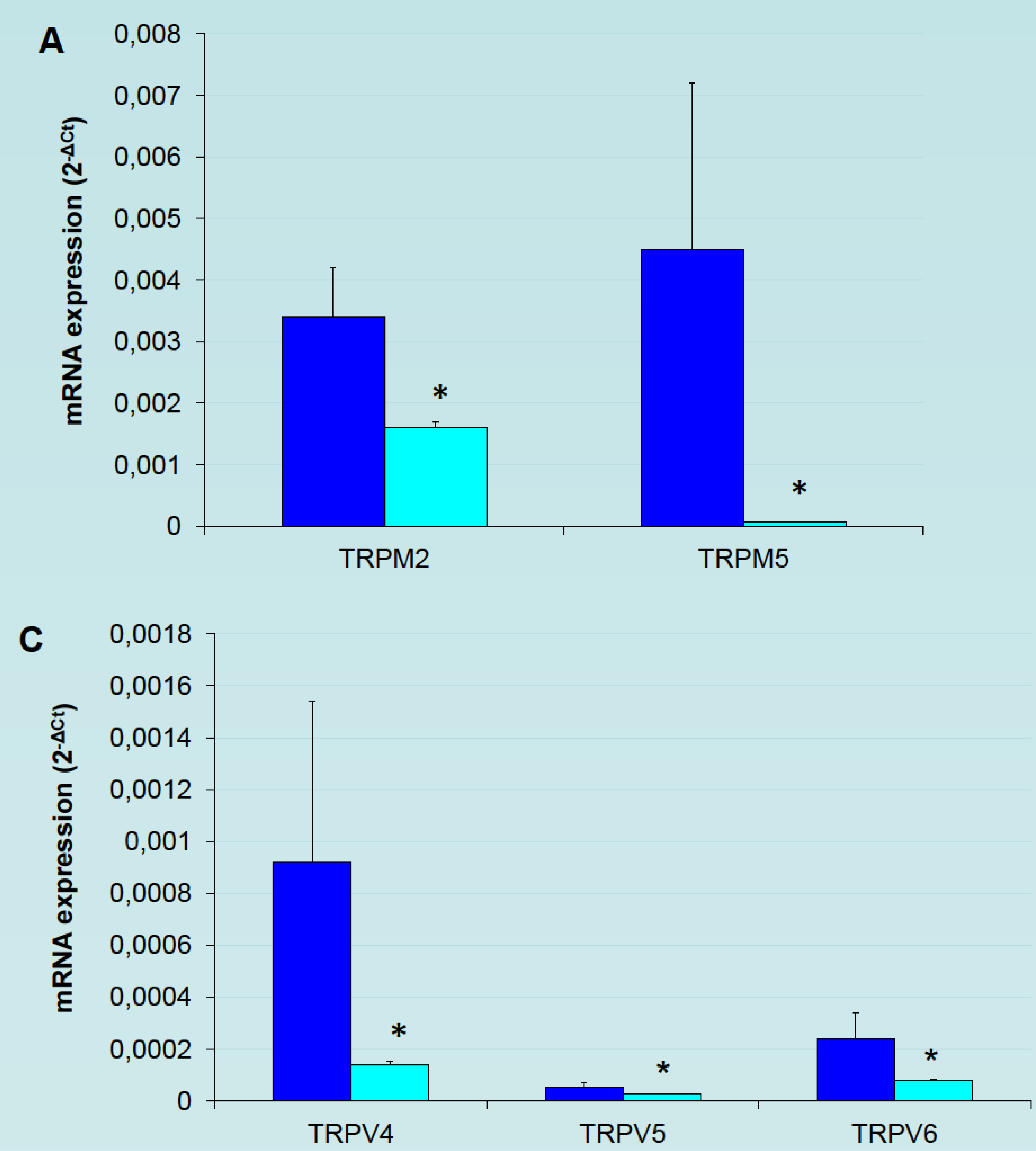

Figure 1. Comparison of the peripheral blood mRNA TRPM2, TRPM5 (A), TRPC1, TRPC3, TRPC6 (B), TRPV4, TRPV5, TRPV6 (C), MCOLN2 (TRPML2), and MCOLN3 (TRPML3) (D) expressions in healthy controls ( $\mathrm{n}=41$, dark blue bars) and in patients with metabolic syndrome $\left(\mathrm{n}=54\right.$, light blue bars). Values are given as mean $\pm \mathrm{SEM},{ }^{*} \mathrm{P}=0.0004, \mathrm{P}<0.0001, \mathrm{P}=0.0082, \mathrm{P}=0.0025$, $\mathrm{P}=0.0035, \mathrm{P}=0.0326, \mathrm{P}=0.0079, \mathrm{P}=0.0003, \mathrm{P}=0.0029$, and $\mathrm{P}<0.0001$ values were obtained for TRPM2, TRPM5 (A), TRPC1, TRPC3, TRPC6 (B), TRPV4, TRPV5, TRPV6 (C), MCOLN2 (TRPML2), and MCOLN3 (TRPML3), respectively.

Gene expression analysis showed that TRPM2, TRPM5 mRNA contents in leukocytes were markedly depressed in MetS patients when compared to the control groups $(\mathrm{P}<0.05$, Figure 1A). There were also marked reductions in TRPC1, TRPC3, TRPV4, TRPV5, TRPV6, MCOLN2 (TRPML2), and MCOLN3 (TRPML3) gene expressions in MetS $(\mathrm{P}<0.05$, Figures 1B-D). Only significant increase was observed with TRPC6 gene expression in patients $(\mathrm{P}=0.0035$, Figure 1B). However, no significant changes in expressions were found with TRPA1, TRPC4, TRPC5, TRPC7, TRPM1, TRPM3, TRPM4, TRPM6, TRPM7, TRPM8, TRPV1, TRPV2, TRPV3, MCOLN1 (TRPML1), and PKD2 (TRPP2) genes in MetS patients $(\mathrm{P}>0.05)$.

This study revealed that there is a statistically significant relationship between TRP channels gene expressions and MetS. Our data showed that TRP channel gene expressions may contribute to the pathology of MetS. Our data also suggest that TRP channels may be a promising future therapeutic target in the treatment of MetS. 